ITM Web of Conferences 4, 01007 (2015)

DOI: $10.1051 /$ itmconf/20150401007

(C) Owned by the authors, published by EDP Sciences, 2015

\title{
Visceral leishmania model
}

Fatima Boukhalfa ${ }^{\mathrm{a}}$, Mohamed Helal ${ }^{\mathrm{b}}$, and Abdelkader Lakmeche ${ }^{\mathrm{c}}$

Laboratory of Biomathematics, Univ. Sidi Bel Abbes, PB. 89, Sidi Bel Abbes 22000, Algeria

\begin{abstract}
In this work we consider a mathematical model based on a system of ordinary differential equations describing the evolution of population of dogs infected by leishmania diseases. By analyzing the corresponding characteristic equations, the local stability of infection free equilibrium point and infection equilibrium point are discussed. It is shown that if the basic reproduction number $R_{0}$ is less than one, the infection free equilibrium is locally asymptotically stable, whereas if the basic reproduction number $R_{0}$ is great than one the infection equilibrium point is locally asymptotically stable, and the infection free equilibrium is unstable.
\end{abstract}

\section{Introduction}

Mathematical modeling of phenomena from biology and medicine are very important to understand and find control methods in order to stop or at least reduce negative effect of diseases (see [1-7]). In this work, we are interested by a model of Zoonotic visceral leishmaniasis (ZVL) developed in [7].

Zoonotic visceral leishmaniasis (ZVL) are caused by infection with leishmania chagasi and is usually transmitted by the sandfly Lutzomyia longipalpis. ZVL is a major public health problem, particularly in Brazil, domestic dogs are the principal reservoir hosts, the biological analysis is discussed in [4].

In this work, we will study the local dynamics, it is enable to obtain the local asymptotic stability of the infection free equilibrium point and the infection equilibrium point when the total of dog population is constant. We consider the model proposed in [4] where the total number of dogs $D$ is divided into everinfectious dogs (that become infectious) and never infected dogs. Ever infectious dogs can be uninfected $S$, latent (infected but not infectious $L$ ), or infectious $I$, never infectious dogs are either uninfected $R$ or

\footnotetext{
a e-mail: boukhalfa_tema@yahoo.fr

b e-mail: mhelal_abbes@yahoo.fr

c e-mail: lakmeche@yahoo.fr
}

This is an Open Access article distributed under the terms of the Creative Commons Attribution License 4.0, which permits unrestricted use, distribution, and reproduction in any medium, provided the original work is properly cited. 
infected $Q$, governed by the following system of ordinary differential equations

$$
\left\{\begin{array}{l}
\frac{d S}{d t}=\alpha \beta D-\frac{C I S}{D}-\delta S, \\
\frac{d L}{d t}=\frac{C I S}{D}-(\sigma+\delta) L \\
\frac{d I}{d t}=\sigma L-\delta I, \\
\frac{d R}{d t}=(1-\alpha) \beta D-\frac{C I R}{D}-\delta R \\
\frac{d Q}{d t}=\frac{C I R}{D}-\delta Q
\end{array}\right.
$$

with the initial conditions

$$
S(0) \geq 0, L(0) \geq 0, I(0) \geq 0, R(0) \geq 0, Q(0) \geq 0 .
$$

The parameters $\alpha, \beta, \delta, C$ and $\sigma$ are positive, where $\alpha$ is the proportion of dogs born susceptible, $\beta$ is the $\operatorname{dog}$ birth rate, $C$ is vectorial capacity, $\delta$ is the dog death rate and $\sigma$ is the rate at which latents $\operatorname{dogs}$ become infectious.

\section{Existence, positivity and boudedness of solution}

\subsection{Existence}

Let $u=(S, L, I, R, Q)^{T}$. The system (1) become

$$
\left\{\begin{array}{l}
u^{\prime}(t)=g(u(t)), \\
u(0)=\left(S_{0}, L_{0}, I_{0}, R_{0}, Q_{0}\right)^{T},
\end{array}\right.
$$

with

$$
g(u)=\left(\begin{array}{c}
\alpha \beta D-\frac{C I S}{D}-\delta S \\
\frac{C I S}{D}-(\sigma+\delta) L \\
\sigma L-\delta I \\
(1-\alpha) \beta D-\frac{C I R}{D}-\delta R \\
\frac{C I R}{D}-\delta Q
\end{array}\right) .
$$

The function $g$ is locally Lipschitz, by Cauchy-Lipschitz theorem, we obtain the local existence of solutions. 


\section{WMLS 2014}

\subsection{Positivity}

Since the right hand sides of (1) are polynomial, system (1) is quasipositive, if the condition

$$
u \geq 0, \quad u_{k}=0 \Rightarrow g_{k}(u) \geq 0,
$$

where $g=\left(g_{1}, \ldots, g_{k}\right)$ for all $k=1, \ldots, 5$.

Hence solutions of (1), (2) with initial conditions $u(0) \in R_{+}^{5}$ stay in $R_{+}^{5}$ for all positive times.

\subsection{Boundedness}

First, we show that the solution of system (1) is bounded.

Let $(S(t), L(t), I(t), R(t), Q(t))$ be a solution of (1) with initial conditions (2), and $[0, T)$ be the maximal interval of existence of the solution.

Let

$$
D(t)=S(t)+L(t)+I(t)+R(t)+Q(t)
$$

then

$$
\frac{d D}{d t}=\frac{d S}{d t}+\frac{d L}{d t}+\frac{d I}{d t}+\frac{d R}{d t}+\frac{d Q}{d t}
$$

We obtain

$$
\frac{d D}{d t}=(\beta-\delta) D(t)
$$

Then, we have

$$
D(t)=D(0) e^{(\beta-\delta) t} .
$$

For $\beta \leq \delta$, it follows that the total population of dogs is bounded and $0<D(t) \leq D(0)$.

\section{The basic reproduction number of (1), (2)}

Let $f$ be the rate of appearance of new infections in each infected compartment, and $v$ be the rate of transfer of individuals into and out of each infected compartment.

The infected compartment are $L$ and $I$.

Hence,

$$
f(L, I)=\left(\begin{array}{c}
\frac{C I S}{D} \\
0
\end{array}\right)
$$

and

$$
v(L, I)=\left(\begin{array}{c}
-(\sigma+\delta) L \\
\sigma L-\delta I
\end{array}\right) .
$$

We denote by $F$ and $V$ the derivatives of $f$ and $v$ with respect to $(L, I)$, then we have

$$
F\left(E^{*}\right)=\left(\begin{array}{cc}
0 & \frac{C S^{*}}{D} \\
0 & 0
\end{array}\right)
$$

and

$$
V\left(E^{*}\right)=\left(\begin{array}{cc}
-(\sigma+\delta) & 0 \\
\sigma & -\delta
\end{array}\right) .
$$


The basic reproduction number is defined by $R_{0}=\rho\left(-F V^{-1}\left(E^{*}\right)\right)$, where $E^{*}$ is the disease equilibrium. Thus,

$$
-F V^{-1}\left(E^{*}\right)=\left(\begin{array}{cc}
\frac{C \alpha \beta \sigma}{\delta^{2}(\sigma+\delta)} & \frac{C \alpha \beta}{\delta^{2}} \\
0 & 0
\end{array}\right) .
$$

Hence, $R_{0}$ is given by

$$
R_{0}=\frac{C \alpha \beta \sigma}{\delta^{2}(\sigma+\delta)}
$$

\section{Equilibria}

In the following, we suppose that $\beta=\delta$, then (1) is reduced to

$$
\left\{\begin{array}{l}
\frac{d S}{d t}=\alpha \delta D-\frac{C I S}{D}-\delta S \\
\frac{d L}{d t}=\frac{C I S}{D}-(\sigma+\delta) L \\
\frac{d I}{d t}=\sigma L-\delta I \\
\frac{d R}{d t}=(1-\alpha) \delta D-\frac{C I R}{D}-\delta R \\
\frac{d Q}{d t}=\frac{C I R}{D}-\delta Q
\end{array}\right.
$$

In this case, the total population $D$ is constant.

Theorem 4.1: Let $\beta=\delta$. Then the system (3) has a unique trivial equilibrium $E_{f}=(\alpha D, 0,0$, $(1-\alpha) D, 0)$ namely the disease free equilibrium. In addition, for $R_{0}>1$, system (3) admits another nontrivial equilibrium $E^{*}$ which we call the disease equilibrium.

Proof: According to the system (3), the equilibrium points satisfy

$$
\left\{\begin{array}{l}
\alpha \delta D-\frac{C I S}{D}-\delta S=0 \\
\frac{C I S}{D}-(\sigma+\delta) L=0 \\
\sigma L-\delta I=0 \\
(1-\alpha) \delta D-\frac{C I R}{D}-\delta R=0 \\
\frac{C I R}{D}-\delta Q=0
\end{array}\right.
$$

(i) For $I=0$, the infection free equilibrium point is given by:

$$
E_{f}=\left(S^{0}, L^{0}, I^{0}, R^{0}, Q^{0}\right)=(\alpha D, 0,0,(1-\alpha) D, 0)
$$




\section{WMLS 2014}

This equilibrium corresponds to a state in which there are no infectious dogs and thus complete eradication of the disease.

(ii) For $I \neq 0$, we show that the system (3) admits another infection equilibrium point $\left(S^{*}, L^{*}, I^{*}, R^{*}, Q^{*}\right)=\left(\frac{\delta D(\sigma+\delta)}{C \sigma}, \frac{\alpha \delta D}{\sigma+\delta}-\frac{\delta^{2} D}{\sigma C}, \frac{\sigma \alpha \beta D}{\delta(\sigma+\delta)}-\frac{\delta D}{C},(1-\alpha) \frac{D \delta(\sigma+\delta)}{C \alpha \sigma},(1-\alpha)\left(D-\frac{D \delta(\sigma+\delta)}{C \alpha \sigma}\right)\right)$ if and only if $\frac{\alpha C \sigma}{\delta(\sigma+\delta)}>1$, corresponding to $R_{0}>1$.

Hence, the system (3) has an infection equilibrium point $E^{*}=\left(S^{*}, L^{*}, I^{*}, R^{*}, Q^{*}\right)$ if $R_{0}>1$.

\section{Local stability of equilibria}

To examine the local asymptotic stability of these equilibria we compute their linearizations, we analyze the stability for $R_{0}<1$ and $R_{0}>1$.

Theorem 5.1: For system (3)

(i) If $R_{0}<1$, the infection free equilibrium $E_{f}$ is locally asymptotically stable. If $R_{0}>1$, then $E_{f}$ is unstable.

(ii) If $R_{0}>1$, then $E^{*}$ is locally asymptotically stable.

Proof: The linearization matrix of the system (3) is

$$
\mathcal{J}_{(S, L, I, R, Q)}=\left(\begin{array}{ccccc}
-\frac{C I}{D}-\delta & 0 & -\frac{C S}{D} & 0 & 0 \\
\frac{C I}{D} & -(\sigma+\delta) & \frac{C S}{D} & 0 & 0 \\
0 & \sigma & -\delta & 0 & 0 \\
0 & 0 & -\frac{C R}{D}-\frac{C I}{D}-\delta & 0 \\
0 & 0 & \frac{C R}{D} & \frac{C I}{D} & -\delta
\end{array}\right) .
$$

(i) At the infection free equilibrium $E_{f}=\left(S^{0}, L^{0}, I^{0}, R^{0}, Q^{0}\right)$, we obtain the linearization

$$
\mathcal{J}\left(E_{f}\right)=\left(\begin{array}{ccccc}
-\delta & 0 & -C \alpha & 0 & 0 \\
0 & -(\sigma+\delta) & C \alpha & 0 & 0 \\
0 & \sigma & -\delta & 0 & 0 \\
0 & 0 & -(1-\alpha) C & -\delta & 0 \\
0 & 0 & (1-\alpha) C & 0 & -\delta
\end{array}\right) .
$$

The characteristic equation at $E_{f}$ is

$$
(-\delta-\lambda)^{3}\left[\lambda^{2}+(2 \delta+\sigma) \lambda+\sigma \delta+\delta^{2}-\sigma C \alpha\right]=0 .
$$

The eigenvalue $\lambda=-\delta$ has multiplicity 3 , we have

$$
\lambda_{1}+\lambda_{2}=-\sigma-2 \delta<0
$$

and

$$
\lambda_{1} \lambda_{2}=\sigma \delta+\delta^{2}-\sigma C \alpha=\delta(\sigma+\delta)\left(1-R_{0}\right)
$$


Then all five eigenvalues of $\mathcal{J}\left(E_{f}\right)$ are negatives if $R_{0}<1$. By the principle of linearized stability we see that the infection free equilibrium point $E_{f}$ is locally asymptotically stable.

For $R_{0}>1$, we have one positive eigenvalue, then the infection free equilibrium point $E_{f}$ is unstable. This model has threshold parameter, known as the basic reproduction number $R_{0}$, such that if $R_{0}<1$, then the $E_{f}$ is locally asymptotically stable, and the disease can not invade the population, but if $R_{0}>1$, then the $E_{f}$ is unstable and invasion is possible.

(ii) The linearization matrix of the system (3) at $E^{*}=\left(S^{*}, L^{*}, I^{*}, R^{*}, Q^{*}\right)$ is

$$
\mathcal{J}\left(E^{*}\right)=\left(\begin{array}{ccccc}
-\frac{C I^{*}}{D}-\delta & 0 & -\frac{C S^{*}}{D} & 0 & 0 \\
\frac{C I^{*}}{D} & -(\sigma+\delta) & \frac{C S^{*}}{D} & 0 & 0 \\
0 & \sigma & -\delta & 0 & 0 \\
0 & 0 & -\frac{C R^{*}}{D} & -\frac{C I^{*}}{D}-\delta & 0 \\
0 & 0 & \frac{C R^{*}}{D} & \frac{C I^{*}}{D} & -\delta
\end{array}\right) .
$$

The characteristic equation at $E^{*}$ is given by

$$
(\lambda+\delta)\left(\frac{C I^{*}}{D}+\delta+\lambda\right)\left[\lambda^{3}+\left(\frac{C \alpha \sigma}{\sigma+\delta}+2 \delta+\sigma\right) \lambda^{2}+\frac{2 \delta+\sigma}{\sigma+\delta} C \alpha \sigma \lambda+C \alpha \delta \sigma-\delta^{2}(\sigma+\delta)\right]=0 .
$$

Since the conditions of Routh-hurwitz criterion, we have

$$
\begin{gathered}
a_{1}=\frac{C \alpha \sigma}{\sigma+\delta}+2 \delta+\sigma>0, \\
a_{2}=\frac{2 \delta+\sigma}{\sigma+\delta} C \alpha \sigma>(2 \delta+\sigma) \delta>0, \\
a_{3}=C \alpha \delta \sigma-\delta^{2}(\sigma+\delta)>0
\end{gathered}
$$

and

$$
\begin{aligned}
a_{1} a_{2}-a_{3} & >\left[\frac{C \alpha \sigma}{\sigma+\delta}+2 \delta+\sigma\right](2 \delta+\sigma) \delta-C \alpha \delta \sigma+\delta^{2}(\sigma+\delta) \\
& >\frac{C \alpha \delta \sigma(\delta+\delta+\sigma)}{\sigma+\delta}+\delta(2 \delta+\sigma)^{2}-C \alpha \delta \sigma+\delta^{2}(\sigma+\delta) \\
& >\frac{\delta^{2} C \alpha \sigma}{(\sigma+\delta)}+\delta(2 \delta+\sigma)^{2}+\delta^{2}(\sigma+\delta) .
\end{aligned}
$$

That is $a_{1} a_{2}-a_{3}>0$.

Therefore, the infection equilibrium point $E^{*}$ is locally asymptotically stable if it is biologically meaningful, i.e. if $R_{0}>1$. 


\section{WMLS 2014}

\section{Concluding remarks}

In this work we have studied leishmaniases model considered in [4], we have analyzed the stability of infection free equilibrium and infection equilibrium points with respect to the basic reproduction number $R_{0}$ in the case $\delta=\beta$.

It is important to see global stability and to give numerical simulations to illustrate the results obtained. Overwise, it is interesting to see model with control of the diseases.

\section{References}

[1] P. Auger, C. Lett and J. C. Poggiale, Modélisation mathématique en écologie, Dunod, Février 2010.

[2] O. Diekmann and J.A.P. Heesterbeek, Mathematical epidemiology of infectious diseases: model building, analysis and interpretation, Wiley, 2002.

[3] O. Diekmann, J.A.P. Heesterbeek and J.A.J. Metz, On the definition and the computation of the basic reproduction ratio $R_{0}$ in model for infectious diseases in heterogeneous population, J. Math. Biol., 28 (1990) 365-382.

[4] C. Dye, The logic of visceral leishmaniasis control, Am. J. Trop. Med. Hyg. 55 (1996) 125-130.

[5] H. W. Hetcot, The mathematical of infectious diseases. SIAM Rev, 42 (2000) 4, 399-653.

[6] H.W. Hethcote and P. van den Driessch, Some epidemiological model with nonlinear incidence, J. Math. Bio., 29 (1991) 271-287.

[7] P. Van Den Driessche and J. Watmough, Reproduction and sub-threshold endemic equilibria for compartemental models of disease transmission, Math. Biosci., 180 (2002) 1-2, 29-48. 